\title{
A malignant mutation
}

Although integrin polymorphisms have been described in several cancers, determining their physiological relevance can be challenging. To address this problem, a mouse model has now been developed to investigate the effects of a tumourassociated integrin mutation in vivo.

Fiona Watt and colleagues previously identified a constitutive activating mutation in the $\beta 1$ integrin subunit (T188I $\beta 1$ ) that was associated with human squamous cell carcinoma (SCC). In order to study the contribution of this mutation to tumour development, this group have now overexpressed the T188I $\beta 1$ or the wild-type $\beta 1$ subunit in the basal layer of mouse epidermis using the keratin 14 promoter. Transgenic cells with the T188I $\beta 1$ mutation showed increased Erk-MAPK signalling, cell spreading and actin cytoskeletal assembly compared with non-transgenic cells or cells from mice overexpressing wild-type $\beta 1$. However, the T188I $\beta 1$ mutation did not affect epidermal proliferation, epidermal organization or stem cell number. The authors therefore concluded that the T188I $\beta 1$ mutation does not affect normal skin architecture or epidermal homeostasis.

Watt and co-workers then went on to test the effect of the T188I $\beta 1$ mutation in carcinogenesis by treating transgenic and control mice with DMBA, which induces $\underline{H R A S}$ mutations, and TPA, which promotes tumour formation. This two-step treatment induced papilloma formation in all groups of mice. However, significantly more papillomas were observed in the two transgenic groups.

The authors then examined malignant conversion and found that mice with the T188I $\beta 1$ mutation had more SCCs and developed SCCs earlier than the wild-type $\beta 1$ or nontransgenic mice. The papillomas and SCCs in the T188I $\beta 1$ mice were less differentiated than in mice that carried the

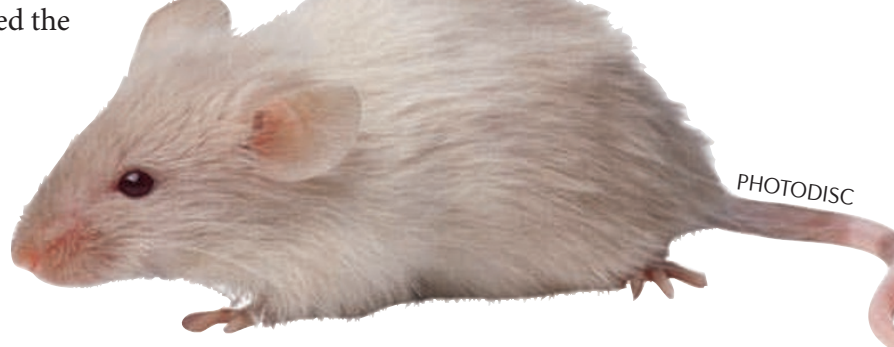

wild-type $\beta 1$ transgene, and showed poor definition between the basal layer and adjacent stroma. Both the wild-type $\beta 1$ and T188I $\beta 1$ variants stimulated lymph node metastasis to a similar extent. The T188I $\beta 1$ papillomas had high levels of phosphorylated Erk, which could contribute to the reduction in their differentiation. These data therefore indicate that integrin mutations may play a part in cancer malignancy and are worthy of further attention.

\section{Meera Swami}

ORIGINAL RESEARCH PAPER Ferreira M. et al. An activating $\beta 1$ integrin mutation increases the conversion of benign to malignant skin tumors. Cancer Res. 69, 1334-1342 (2009) 